\title{
Value of dynamic and DWI MRI in evaluation of HCC viability after TACE via LI-RADS v2018 diagnostic algorithm
}

\author{
Tamer Yousef Saleh $^{1 *}$ DD, Suzan Bahig ${ }^{2}$, Naglaa Shebrya ${ }^{2}$ and Aya Yassin Ahmed ${ }^{2}$
}

\begin{abstract}
Background: Detection of the value of DCE (dynamic contrast enhanced) MRI and DWI (diffusion-weighted imaging) in follow-up of treatment response for HCC (hepatocellular carcinoma) lesions post TACE (trans-arterial chemo-embolization) through LI-RADS v2018 algorithmic approach.

Results: A prospective study was performed on 30 patients with 41 treated hepatic focal lesions. The patients underwent DCE MRI with DWI in less than 1-month duration following TACE procedure and were radiologically assessed to observe tumoral post treatment response; then, another follow-up after another 3 months later was done for non-viable, equivocal, and non-evaluable post treatment response categories. Statistical analysis showed that DCE MRI had $100 \%$ level of sensitivity, specificity of $95.24 \%$, PPV of $95.00 \%$, and NPV of $100 \%$ with an overall agreement of $97.50 \%$. While on the other hand, statistics showed that DWI has $52.63 \%$ level of sensitivity, specificity of $90.48 \%$, PPV of $83.33 \%$, PPV of $67.86 \%$, and NPV of $72.50 \%$.

The difference between non-viable and viable groups' ADC variables was found statistically significant at $P$ value $<$ 0.001 , and best cut off value that augments sensitivity and specificity is 1.35 . At this ADC value, sensitivity reaches $78.9 \%$ and specificity is $85.7 \%$.

Conclusions: Our study showed that DCE MRI has 100\% level of sensitivity via application of LI-RADS v2018 diagnostic algorithmic approach while DWI alone has 52.63\% level of sensitivity, yet it can enhance the diagnostic confidence of DCE MRI for post treatment response viability detection.
\end{abstract}

Keywords: HCC, TACE, DCE MRI, DWI, LI-RADS v2018 treatment response

\section{Background}

Hepatocellular carcinoma was found to be the 4th leading cause of cancer-related deaths during 2018 and being also considered worldwide as the 6th most common malignancy [1]. In oncology, due to the difficultness and accuracy of liver biopsy (up to $40 \%$ false-negative results in lesions $\leq 2 \mathrm{~cm}$ ), non-invasive diagnosis can be achieved with imaging in high-risk populations [2].

DCE MRI is a well-established liver imaging modality via providing accurate qualitative and quantitative data through its multi-parametric capabilities [3].

Guided by the Barcelona Clinic Liver Cancer (BCLC) classification system, trans-arterial chemoembolization

\footnotetext{
* Correspondence: tamer100@gmail.com

${ }^{1}$ Military Medical Academy, Heliopolis, Egypt

Full list of author information is available at the end of the article
}

(TACE) is the first line of treatment for HCC patients with intermediate stage and those with sizable or multicentric HCC [4]. Follow-up for the effectiveness of TACE with imaging is essential in evaluating the outcome of TACE and the next future step along the treatment plan of the patient [5].

It is often hard to assess enhancement of contrast in a treated lesion with partial retention of lipidol droplets through CT studies, due to the inevitable beam hardening imaging artifacts of lipidol droplets. On the other hand, it was found that MRI signals are not degraded by lipidol droplets; therefore, a residual/newly developed lesion is better detected by DCE MRI. In this manner, DCE MRI study for the hepatic lesions is viewed now as the chosen radiological modality [5].

Liver Imaging Reporting and Data Systems (LI-RADS) started introducing the concept of LR-treated in v2014; 
then, another new treatment response algorithm was made and published in the 2017 update for radiologists' assistance in different imaging modality interpretation for HCC following loco-regional therapy. In addition to offering imaging criteria for viable and nonviable treated HCC lesions, new categories of non-evaluable lesions as well as lesions with equivocal tumoral viability were introduced through application of major and other ancillary imaging features [6].

Following trans-catheter therapies (TACE, DEBTACE, or TAE), different forms of post treatment patterns of response emerged. In general, in the LR-TR viable post treatment category response for TACE treatment, dynamic MRI shows APHE in the form of either nodular thick or mass-like area following contrast administration which may be mixed within necrotic regions. The peri-lesional enhancement detected through dynamic MRI is not pathognomonic for the recurrent lesions [7], but also can occur in non-viable conditions due to adjacent inflammatory infiltrate or other nontumoral arterio-portal shunts as in the case of direct arterio-portal fistula or reduced portal venous flow caused by iatrogenic induced portal tract injuries in the vicinity of the TACE procedure and by later time it resolves [8].

DWI can give data about tumor viability degree. Nonviable treated hepatic lesions show less hyper-intense signals in DWI with corresponding increase in ADC mapping series values as a result of cell membrane damage. On the other hand, viable treated hepatic focal lesions still show diffusion restriction and relatively low $A D C$ values. $A D C$ mapping series give idea about intraand extracellular water molecule movement in biological tissue and hence gives data about the tumoral microenvironment [7].

Diffusion-weighted imaging has a great value in detection of areas with tumoral viability tumor; however, it does not replace identification of characteristic enhancement patterns of tumoral viability through dynamic study that is why it is currently not included in the LIRADS v2018 treatment response algorithm as a major feature [9].

\section{Methods}

\section{Study population}

Inclusion criteria

- A prospective study performed on patients who underwent TACE referred from the Tropical and Gastroenterology Departments to Radio-diagnosis Department. All patients had cirrhotic liver due to chronic viral hepatitis. All patients signed written consents. The study is IBR (institutional board review) approved.

\section{Exclusion criteria}

- Systemic chemotherapy treatment for HCC

- Other liver mass lesions other than hepatocellular carcinoma

- MRI contraindications, e.g., claustrophobia and cardiac pacemakers

- Contraindications to administration of contrast media

\section{Methods}

All cases had been subjected to the following:

- Full clinical assessment

- Revision of the patient's renal function investigations including blood urea and serum creatinine tests

- Revision of the prior radiological investigations for the patients

All patients come in for follow-up by DCE MRI with diffusion-weighted images in less than 1-month duration following TACE procedure to observe tumoral post treatment response to TACE; then, another follow-up after another 3 months later was done for non-viable, equivocal, and non-evaluable post treatment response categories. While For LR-TR viable lesions, diagnosis was made depending on the 1-month/3-month followup MRI findings of major and ancillary feathers or sustained radio-dense iodized oil droplets accumulations in the hyper-vascular lesion/area on the session of hepatic arteriography with next TACE.

\section{Equipment}

DCE MRI with DWI was performed using GE 3 Tesla MRI scanner (Discovery 750).

\section{Techniques}

- Examination included pre-contrast axial and coronal T1-weighted out-of-phase (OP) and in-phase (IP) imaging and T2 in addition to axial SPIR-weighted images followed by diffusion MRI then DCE MRI images were obtained following bolus injection of $0.1 \mathrm{mmol} / \mathrm{kg}$ body weight of gadolinium-DTPA at injection rate of $2 \mathrm{ml} / \mathrm{s}$; then, flushing with $20 \mathrm{ml}$ of sterile saline solution was done through the antecubital vein with a power injector.

- Diffusion imaging was obtained prior to (DCE)-MRI using respiratory gated fat-suppressed single-shot spin-echo echo-planar sequence. It was performed by applying different $b$ values of 20,500, and $800 \mathrm{~s} /$ $\mathrm{mm}^{2}$.

- DCE MRI was obtained using axial T1-weighted FSPGR LAVA (liver acquisition with volume acceleration) contrast-enhanced imaging that was 
performed in triphasic manner; DCE MRI series included one pre contrast series then four successive post contrast series consisting of early arterial, predominant arterial (or late arterial) phase, portal phase, and a delayed (equilibrium) phase with 1720 -s intervals (15-17 s for image acquisition with breath-holding technique and 2-3s for rebreathing) followed by 3-4-min delayed phase imaging. Imaging of all patients was in end of expiration to diminish the risk of image misregistration

\section{Dynamic analysis}

- Size (longest axial dimension through post contrast enhancing area of treated lesion, not traversing nonenhancing area) of the lesion and its border.

- The signal pattern in T1 and T2 as well as fat saturated sequences.

- Enhancement pattern through the DCE MRI and subtracted images where APHE is considered when there is arterial hyper-vascularity (definitely greater than that of background liver) and/or subsequent contrast washout appearance (relative hypo-intensity of the treated lesion in comparison to background liver parenchyma) on the following phases; those were regarded as major features suggestive of LR-TR viable $\mathrm{HCC}$ treated lesions depending on the 1month/3-month follow-up MRI findings of major and ancillary feathers or sustained radio-dense iodized oil droplets accumulations in the hypervascular lesion/area on the session of hepatic arteriography with next TACE. On the other hand, LRTR non-viable post treatment response category was considered when absence of treated lesion enhancement or expected treatment-specific enhancement like thin smooth marginal enhancement or an illdefined geographic regional enhancement along the vicinity of the treated tumor which may be valued as a transient post-procedural perfusion alteration on dynamic images. Yet there is still a fact that radiologic signs of treated lesion non-viability are not equivalent with pathologic complete ablation response, because imaging modalities are not sensitive to small residual tumor foci.

\section{Diffusion sequence interpretation}

- Signal intensity on DWI and ADC mapping series with ADC values calculation using a commercially windows workstation (GE) were categorized according to three scales grading:

1. Any treated hepatic lesion was categorized as restricted if it showed frank obvious higher signals than background hepatic parenchyma on
DWI and/or frank obvious hypo-intense signals than background the liver in ADC.

2. Uncertainty is considered if faint sustained hyper-intensity is noticed on DWI and no corresponding hypo-intensity on ADC maps.

3. Finally, facilitated diffusion is considered if treated lesion showed iso-intense signals in DWI and ADC series or if it lost its pre-procedural restriction or showing T2 shine-through effect.

- ADC value for each treated hepatic lesion was recorded.

- The standard of reference in our study, surgery was not the suitable choice for treatment of our cases, and hence, it was hard to obtain histopathology report for any of the patients in addition to biopsy sampling errors existence owing to technical difficulties, so we depended on the LI-RADS v2018 major and ancillary features were used to evaluate post TACE treatment response. So, our standard of reference was:

- LR-TR viable category (residual/ recurrent HCC) (Figs. 1 and 4): For treated lesions with non-rim peripheral APHE and/or delayed washout in DCE MRI follow-up imaging or sustained lipidol droplets accumulation in the hyper-vascular treated lesion on the hepatic arteriography with another TACE procedure

- LR-TR non-viable (resolved lesions) (Figs. 2 and 3): Treated hepatic lesions with no pathological arterial enhancement or with TACE-specific expected enhancement

- LR-TR non-evaluable category: For cases with image degradation due to motion artifacts

- LR-TR equivocal: Atypical enhancement not expected for treatment-specific expected post contrast enhancement pattern and also not meeting criteria for viability

- Diffusion restriction was also treated as a sign of residual/recurrence detection and promotes reader confidence hand in hand with positive dynamic imaging findings (Fig. 4).

\section{Statistical analysis}

- Data were coded and entered using the statistical package SPSS (Statistical Package for the Social Sciences) version 25 and were summarized using mean, standard deviation, median, minimum and maximum in quantitative data, and frequency (count) and relative frequency (percentage) for categorical data. Standard diagnostic indices including sensitivity, specificity, positive predictive value (PPV), negative predictive value (NPV), and diagnostic efficacy were calculated [10]. 


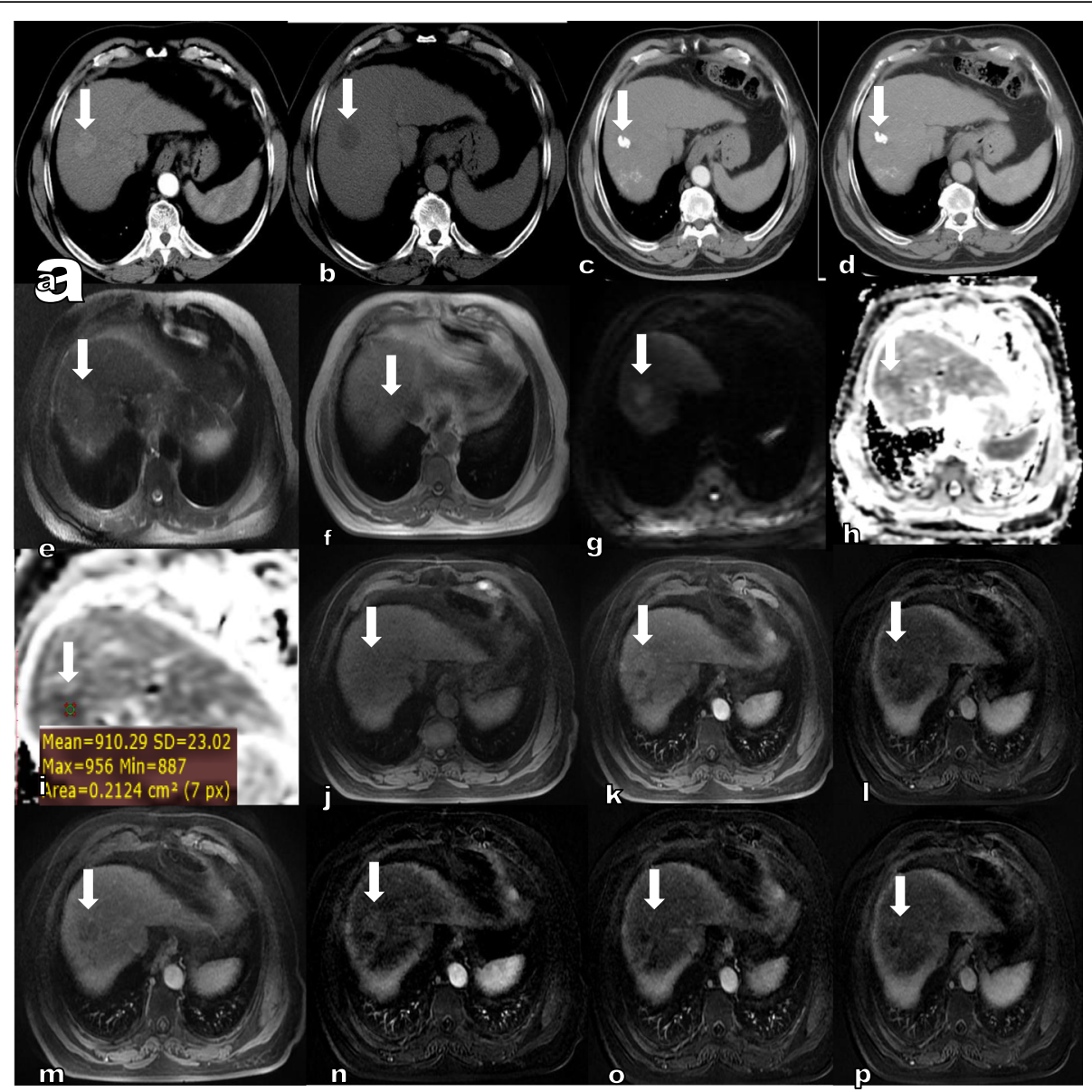

Fig. 1 A 66-year-old male patient having HCC underwent TACE ablation for one focal lesion involving right hepatic lobe segment VIII. a Pre-TACE CT-scan in the arterial phase showing intense enhancement. b Wash-out in delayed phase. c Follow-up with CT-scan showing no definite enhancement in the arterial phase. $\mathbf{d}$ No definite wash-out in delayed phase. e Yet the patient had high AFP level, so DCE MRI was done showing bright signals in T2. $\mathbf{f}$ Relative hypo-intense signals in T1 WIs. $\mathbf{g}$ Restricted diffusion in DWI at 800 b value. $\mathbf{h}$ Corresponding dark signals in $A D C$ mapping series. i ADC value $=0.91 \times 10^{-3} \mathrm{~s} / \mathrm{mm}^{2}$. $\mathbf{j}$ Iso-intense signals in pre-contrast phase of LAVA. $\mathbf{k}$ Arterial enhancement in late arterial phase. I Wash out of the contrast in the porto-venous. $\mathbf{m}$ Delayed phase. $\mathbf{n}$ Being confirmed in subtraction images throughout arterial, $\mathbf{o}$ porto-venous, and $\mathbf{p}$ delayed phases. Final diagnosis: LR-TR viable $2.9 \mathrm{~mm}$, (pretreatment, LR-5, $28 \mathrm{~mm}$ )

- ROC curve was built with area under the curve analysis obtained to detect best cutoff value of ADC measurement for detection of viability.

- Comparisons between quantitative variables were done using the non-parametric Mann-Whitney test [11]. For comparing categorical data, chi-square $\left(\chi^{2}\right)$ test was performed.

- Exact test was used instead when the expected frequency is less than 5 [12]. $P$ value less than 0.05 was considered as statistically significant.

\section{Results}

This study was prospectively carried on 30 patients ( 2 females and 28 males) with 41 treated lesions; the mean age for all patients was 62.63 years (age range, 49-72 years) as shown in Table 1.
Nineteen from the $40 \mathrm{HCC}$ treated lesions showed radiological features suggestive of LR-TR viable post treatment response while on the other hand 21 treated lesions were considered as LR-TR non-viable due to proper tumoral embolization. Our study included one case categorized as non-evaluable due to image motion artifacts, and on follow-up, it turns up to be viable lesion as shown in Fig. 5.

Non-viable treated hepatic lesions' size ranged from 1 to $4.5 \mathrm{~cm}$, and viable treated lesions' size ranged between 1.2 and $7.3 \mathrm{~cm}$ in maximum diameter.

The mean and standard deviation of LR-TR viable and LR-TR non-viable groups are illustrated in Table 2.

Among 40 treated lesions in our study, $45 \%$ of lesions showed heterogeneous T2 signal intensity and $61 \%$ of the treated hepatic lesions showed heterogeneous T1 signal intensity making the evaluation of $\mathrm{HCC}$ post 


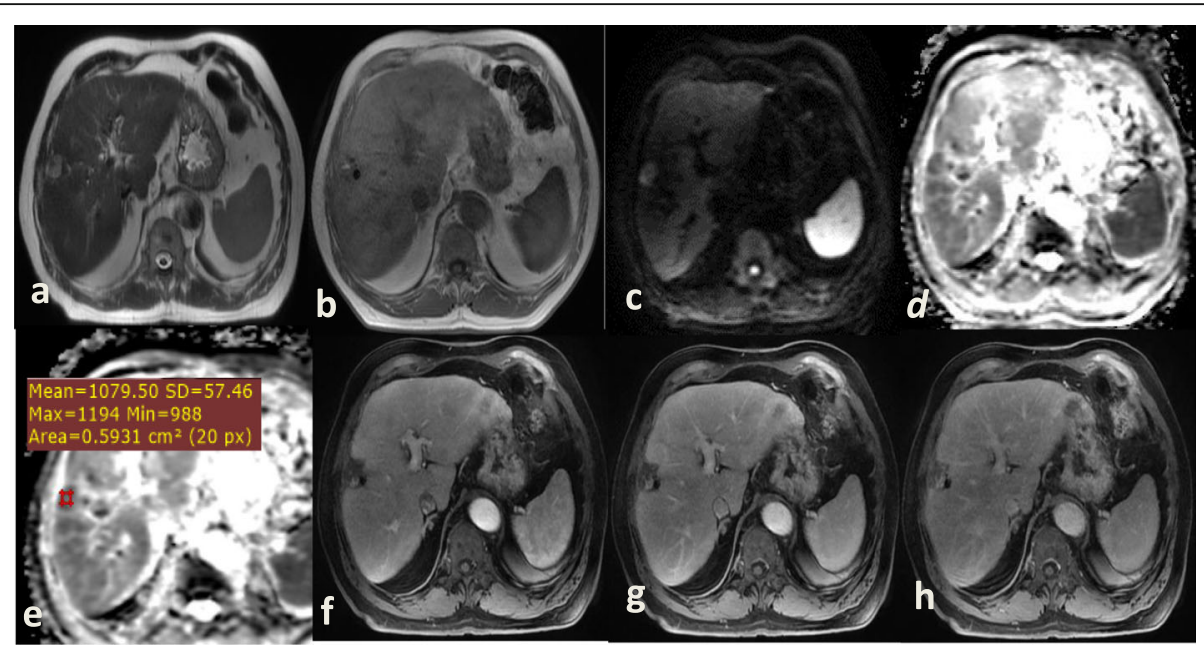

Fig. 2 1st follow-up after 1 month for a 69-year-old male patient having HCC underwent TACE ablation for one focal lesion involving right hepatic lobe segment V. a Heterogeneous signals (predominantly bright) in T2 WIs. b Heterogeneous signals (predominantly iso-intense with hyper-intense and hypo-intense foci) in T1 Wls. $\mathbf{c}$ Restricted diffusion in DWI at 800 b value. $\mathbf{d}$ Corresponding dark signals in ADC mapping series. e $A D C$ value $=1.07 \times 10^{-3} \mathrm{~s} / \mathrm{mm}^{2}$. $\mathbf{f}$ Arterial, $\mathbf{g}$ porto-venous, and $\mathbf{h}$ delayed phases. Small area of progressive enhancement along the posteromedial portion of the hepatic observation representing granulation tissue (expected post TACE response). Final diagnosis: LR-TR non-Viable

TACE response depending on pre-contrast sequences signal intensity a matter of disagreement.

Table 3 shows how the lack of APHE represents good post TACE response in $95.2 \%$ of LR-TR non-viable category (i.e., true negative) in DCE MRI study, while APHE with/or delayed washout represents tumoral viability in $100 \%$ of the LR-TR viable category (i.e., true positive) in DCE MRI study. We had no false-negative results among LR-TR viable category.
Table 4 shows how facilitated lesions on DWI represent excellent therapeutic response of $90.48 \%$ of LR-TR non-viable lesions (i.e., true negative), while around $47.4 \%$ of the LR-TR viable lesions lack diffusion restriction (i.e., false negative) (Fig. 6). On the other hand, $52.6 \%$ of the LR-TR viable lesions showed restricted diffusion (i.e., true positive), while around $9.52 \%$ of the LRTR non-viable lesions showed restricted diffusion (i.e., false positive).

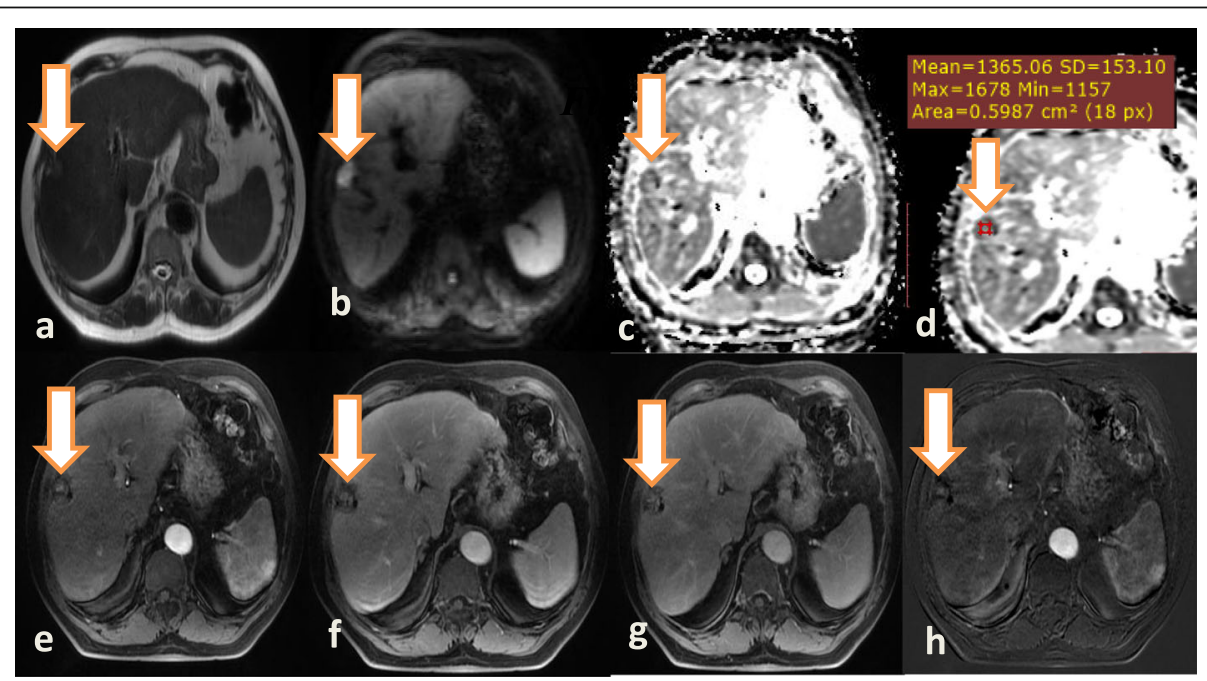

Fig. 3 2nd follow-up after 3 months for a 69-year-old male patient having HCC underwent TACE ablation for one focal lesion involving right hepatic lobe segment V. a Heterogeneous signals (predominantly bright) in T2 WIs. b Restricted diffusion in DWI at 800 b value. c Corresponding dark signals in ADC mapping series. $\mathbf{d}$ ADC value $=1.36 \times 10^{-3} \mathrm{~s} / \mathrm{mm}^{2}$ increased ADC value in comparison to the 1st follow-up. e Late arterial, $\mathbf{f}$ porto-venous, and $\mathbf{g}$ delayed phases. $\mathbf{h}$ Late arterial subtraction images. Small area of progressive enhancement along the posterior portion of the hepatic observation representing granulation tissue (expected post TACE response). Final diagnosis: LR-TR non-viable 


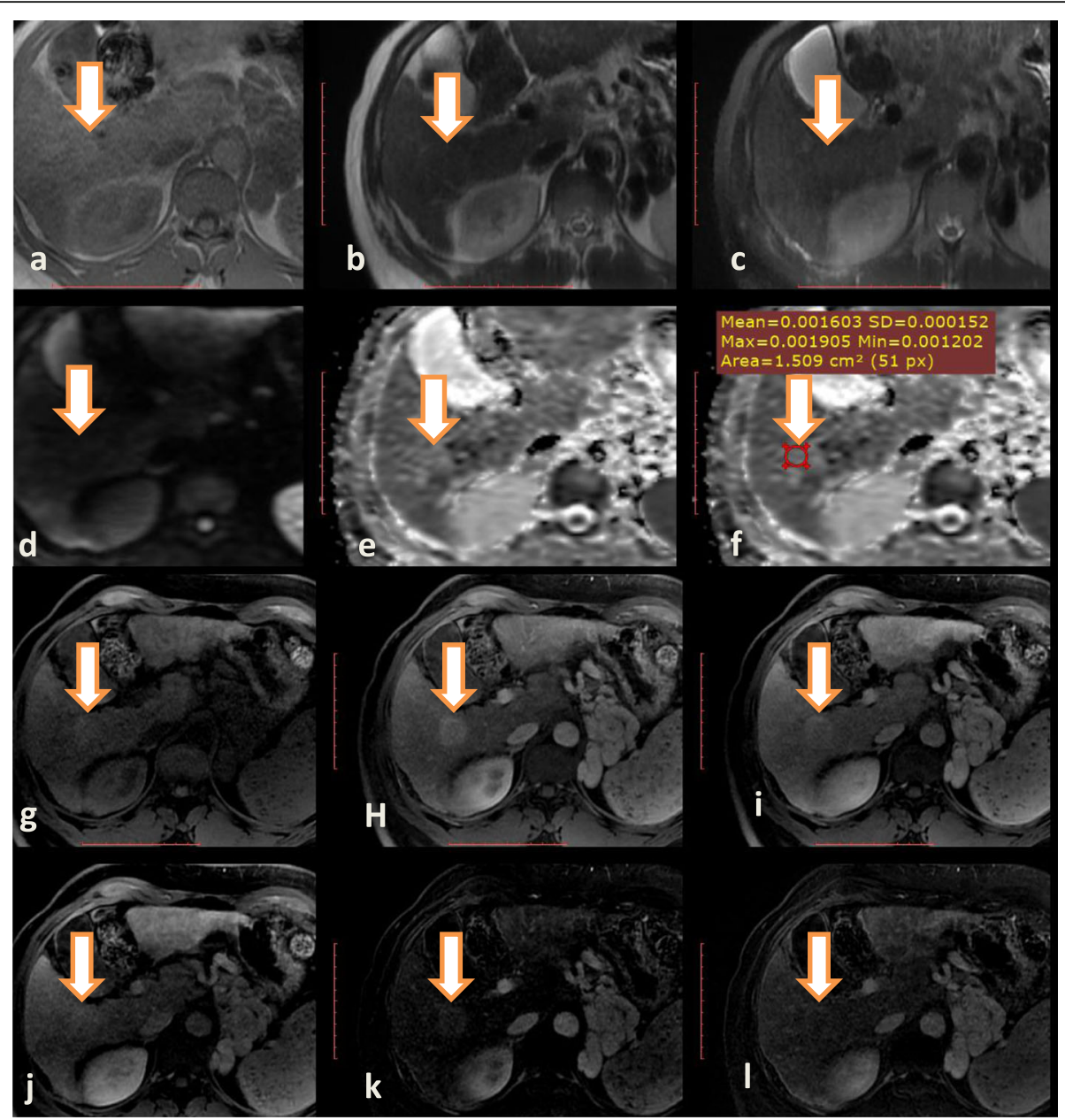

Fig. 4 1st follow-up DCE MRI with DWI after 1 month for a 55-year-old male patient with chronic viral hepatitis C diagnosed as HCC involving right hepatic lobe segment VI underwent TACE ablation with still elevated AFP level. a Treated lesion showed faint high in T1 WIs. b Faint high signals T2 WIs. c Faint high signals STIR Wls. d Facilitated diffusion in DWI at 800 b value. e Corresponding bright signals in ADC mapping series. $\mathbf{f}$ ADC value $=1.60 \times 10^{-3} \mathrm{~s} / \mathrm{mm}^{2}$. g Faint high signal in pre-contrast LAVA sequence. $\mathbf{h}$ Non-rim APHE is seen in late arterial phase. i Porto-venous phase showing faint wash-out. $\mathbf{j}$ Faint wash-out in delayed phase. $\mathbf{k}$ Non-rim APHE is confirmed in late arterial phase with subtraction. I Faint wash-out is confirmed in delayed phase with subtraction. Final diagnosis: LR-TR viable $27 \mathrm{~mm}$

Statistical analysis showed that DCE MRI had 100\% level of sensitivity, specificity of $95.24 \%$, PPV of $95.00 \%$, and NPV of $100 \%$ with an overall agreement of $97.50 \%$. While on the other hand, DWI has $52.63 \%$ level of sensitivity, specificity of $90.48 \%$, PPV of $83.33 \%$, PPV of $67.86 \%$, and NPV of $72.50 \%$.

The difference between the LR-TR viable post TACE response and LR-TR non-viable groups ADC values was statistically significant with a $P$ value of $<$ 0.001 .
The ROC curve obtained by plot at different cut off values is shown in Fig. 7. The best cutoff value that maximizes sensitivity and specificity is 1.35 . At this ADC value, the sensitivity is $78.9 \%$ and specificity is $85.7 \%$. A statistical software showed that the area under the curve is $C=0.861$ with $\mathrm{SE}=0.064$ and $95 \%$ CI from 0.735 to 0.987 .

According to Table 5, DCE MRI had a sensitivity value of $100 \%$, and a specificity of ROC curve showed that $\mathrm{ADC}$ variable is a good indicator to differentiate tumoral

Table 1 The demographic data of 40 treated observations

\begin{tabular}{|c|c|c|c|c|c|c|c|c|c|c|}
\hline & \multicolumn{5}{|c|}{ LR-TR viable } & \multicolumn{5}{|c|}{ LR-TR non-viable } \\
\hline & Mean & SD & Median & Minimum & Maximum & Mean & SD & Median & Minimum & Maximum \\
\hline$\overline{\text { Age }}$ & 62.05 & 4.67 & 63.00 & 53.00 & 70.00 & 63.24 & 6.30 & 65.00 & 49.00 & 72.00 \\
\hline
\end{tabular}




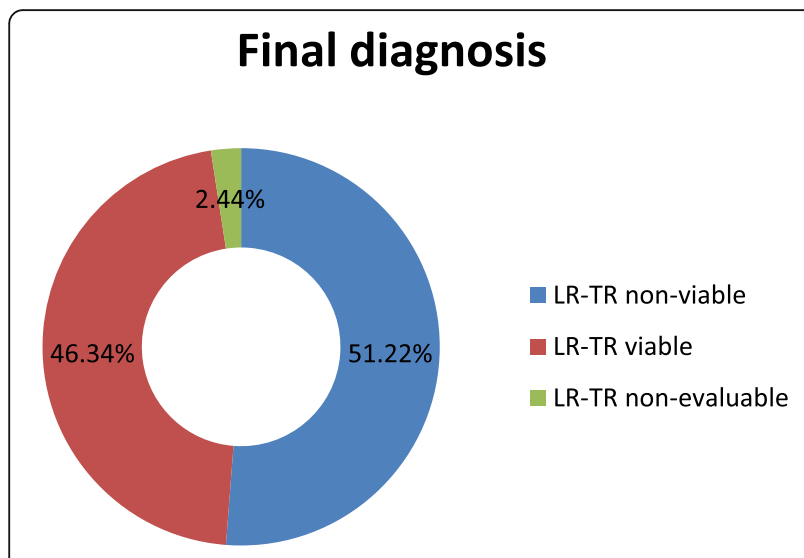

Fig. 5 Distribution of observations into LR-TR viable, LR-TR nonviable, and LR-TR non-evaluable groups

viability for $\mathrm{HCC}$ from perilesional pseudo- or nonviable treated lesions.

\section{Discussion}

We carried out our study for assessment of the value of DCE MRI and DWI in evaluation of post treatment response for TACE as a treatment option for HCC via LIRADS v2018 diagnostic algorithmic approach (Table 6).

DCE MRI examination has a crucial role in assessment of HCC viability following TACE via bypassing the interference of accumulated iodized oil as non-contrast conventional MRI sequences show morphological and fluid content changes as well as fibrosis, while dynamic study clearly show the perfusional criteria of treated lesion [5].

Ebeed et al. [5] and Afifi et al. [13] showed that treated lesions exhibit hyperintensity on conventional T1 WIs and hypo-intensity on conventional T2-WIs images with T2 hyperintensity in cases of viability. Hyper-intense signals on T2 WIs not only represent tumoral viability but also could be seen in case of lesional hemorrhage, inflammation, or cystic necrosis [5, 13]. Among our 41 post TACE hepatic lesions included in our study, about $61 \%$ of lesions demonstrate heterogenous signals in $\mathrm{T} 1$ WIs and $46 \%$ in T2 WIs that make a conflict issue during evaluation of post treatment response after TACE depending on conventional MRI signal intensity on noncontrast basis only.

Through our study, we had higher sensitivity for DCE MRI compared to other researches done by Ebeed et al. [5], Osama et al. [14], Yu et al. [15], and Goshima et al. [16]; this occurs because in all other researches,
Table 3 Correlating DCE MRI results to the final diagnosis in the studied group

\begin{tabular}{|c|c|c|c|c|c|c|c|}
\hline & & \multicolumn{2}{|c|}{ LR-TR viable } & \multicolumn{2}{|c|}{ LR-TR nonviable } & \multicolumn{2}{|c|}{ Total } \\
\hline & & Number & $\%$ & Number & $\%$ & & $\%$ \\
\hline \multirow[t]{5}{*}{ Dynamic results } & TP & 19 & 100 & - & - & 19 & 47.5 \\
\hline & FN & 0 & 0 & - & - & 0 & 0 \\
\hline & FP & - & - & 1 & 4.7 & 1 & 2.5 \\
\hline & $\mathrm{TN}$ & - & - & 20 & 95.3 & 20 & 50 \\
\hline & Total & 19 & 100.0 & 21 & 100.0 & 40 & 100 \\
\hline
\end{tabular}

inappropriate breath holding led to motion artifacts with subsequent false-negative interpretation by the viewer; however, in our study, we had one case that was categorized as LR-TR non-evaluable and follow-up showed that it is viable; therefore, there are no definite falsenegative cases in our study $[5,14-16]$. We also had one false-positive case due to misinterpretation of a perilesional arterioportal enhancement.

Afifi et al. [13] stated that the increase in false-positive findings originated from perilesional parenchymal insults that showed sustaining hyperintensity on DWI with increasing $b$ factors. They demonstrated that hypercellularity intermingled with a fibrotic component in the inflammatory granulation tissue could restrict water diffusion [13].

In the experiences of Ebeed et al. [5] and Osama et al. [14], liquefactive breaking down and intra-lesional necrosis are recognized to be the reason of hyper-intensity in DWI images of post therapeutic breaking down of viable lesions [5, 14].

In our study, two false-positive lesions out of 40 lesions were misdiagnosed on diffusion-weighted imaging. We considered that false-positive findings in our study is likely originating from post treatment hemorrhage or liquefactive tumoral necrosis that causes lack of diffusion facilitation.

Tantawy and Mohamed [17] demonstrated the difference in ADC values in HCC lesions in studies done before and following TACE application. The mean ADC for the lesions before TACE was $1.27 \pm 0.25 \times 10^{-3} \mathrm{~s}$ / $\mathrm{mm}^{2}$ and increased following treatment in responding lesions to reach $1.57 \pm 0.22 \times 10^{-3} \mathrm{~s} / \mathrm{mm}^{2}$ with a statistically significant difference $(P=0.002)$ [17].

In our study, the difference between ADC variables between the viable and non-viable groups was statistically significant with $P$ value $<0.001$. The best cutoff that sensitivity and specificity values are maximized at is 1.35 . At

Table 2 Diameter in cm (40 treated observations)

\begin{tabular}{|c|c|c|c|c|c|c|c|c|c|c|}
\hline & \multicolumn{5}{|c|}{ LR-TR viable } & \multicolumn{5}{|c|}{ LR-TR non-viable } \\
\hline & Mean & SD & Median & Minimum & Maximum & Mean & SD & Median & Minimum & Maximum \\
\hline Size in $\mathrm{cm}$ & 3.74 & 1.42 & 4.00 & 1.20 & 7.30 & 2.56 & 1.03 & 2.40 & 1.00 & 4.50 \\
\hline
\end{tabular}


Table 4 Correlating diffusion MRI results to the final diagnosis in the studied group

\begin{tabular}{|c|c|c|c|c|c|c|c|}
\hline & & \multicolumn{2}{|c|}{ LR-TR viable } & \multicolumn{2}{|c|}{ LR-TR nonviable } & \multicolumn{2}{|c|}{ Total } \\
\hline & & Number & $\%$ & Number & $\%$ & & $\%$ \\
\hline \multirow[t]{5}{*}{ Diffusion MRI (uncertainty) as a positive interpretation } & TP & 9 & 52.6 & - & - & 9 & 22.5 \\
\hline & FN & 10 & 47.4 & - & - & 10 & 25 \\
\hline & FP & - & - & 2 & 9.5 & 2 & 5 \\
\hline & TN & - & - & 19 & 90.5 & 19 & 47.5 \\
\hline & Total & 19 & 100.0 & 21 & 100.0 & 40 & 100.0 \\
\hline
\end{tabular}

this ADC value, the sensitivity is $78 \%$ and specificity is $85 \%$. We found from the ROC that ADC variable is a good indicator to differentiate tumoral viability of HCC from expected post treatment findings seen in nonviable lesions.

Studies done by Ebeed et al. [5], Tantawy and Mohamed [17], proved that ADC mapping series values increased in non-viable lesions after TACE than in viable lesions $[5,17]$.

Realistically speaking, to demonstrate post TACE response whether viable or not is more important than measuring $\mathrm{ADC}$ value before and following TACE. In the experience of $\mathrm{Yu}$ et al. [15], ADC value was used to depict the peripheral tumoral recurrence for HCC from pseudo- or benign expected peri-lesional findings. His study demonstrate results that were not with statistically significant differences; ADC values differed broadly for the non-viable lesions and deeply overlapped with the residual tumoral lesions, and thus, establishing cutoff points was impossible [15].
Our results are equivalent with those of Ebeed et al. [5], Osama et al. [14], and Goshima et al .[16] showing that DCE MRI is to be predominant to DWI in assessing post TACE treatment response and DWI is not continuously a solid indicator of HCC post treatment viability in comparison to DCE MRI $[5,14,16]$. As in our study, DCE MRI had a sensitivity of $100 \%$, a specificity of $95.25 \%$, a positive predictive value of $95 \%$, a negative predictive value of $100 \%$, and overall agreement of $97.5 \%$ while DWI statistics values are 52.63\%, 90.48\%, 83.33\%, $67.86 \%$, and $72.5 \%$ respectively.

Yousef et al. [18] and our study agreed that DWI has some privileges in comparison to dynamic MRI study. For instance, contrast agent administration is contraindicated, and for a relatively shorter time examination. Also, it is simple to repeat the technique, allowing close follow-up throughout and following treatment. Furthermore, less time is consumed in post-processing for DWI images in comparison to DCE MR imaging. Finally, DWI is beneficial in cases of in-homogeneity that may

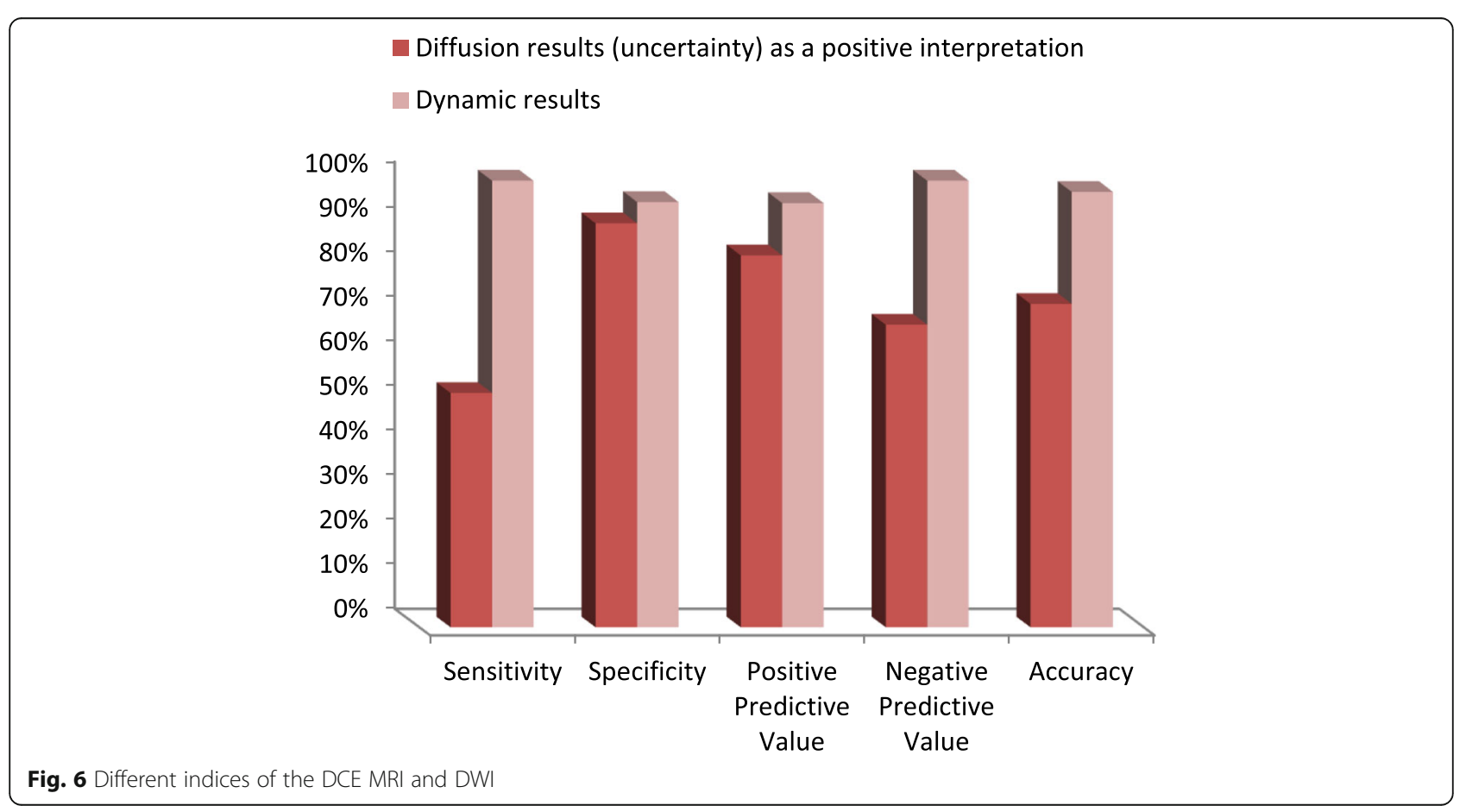




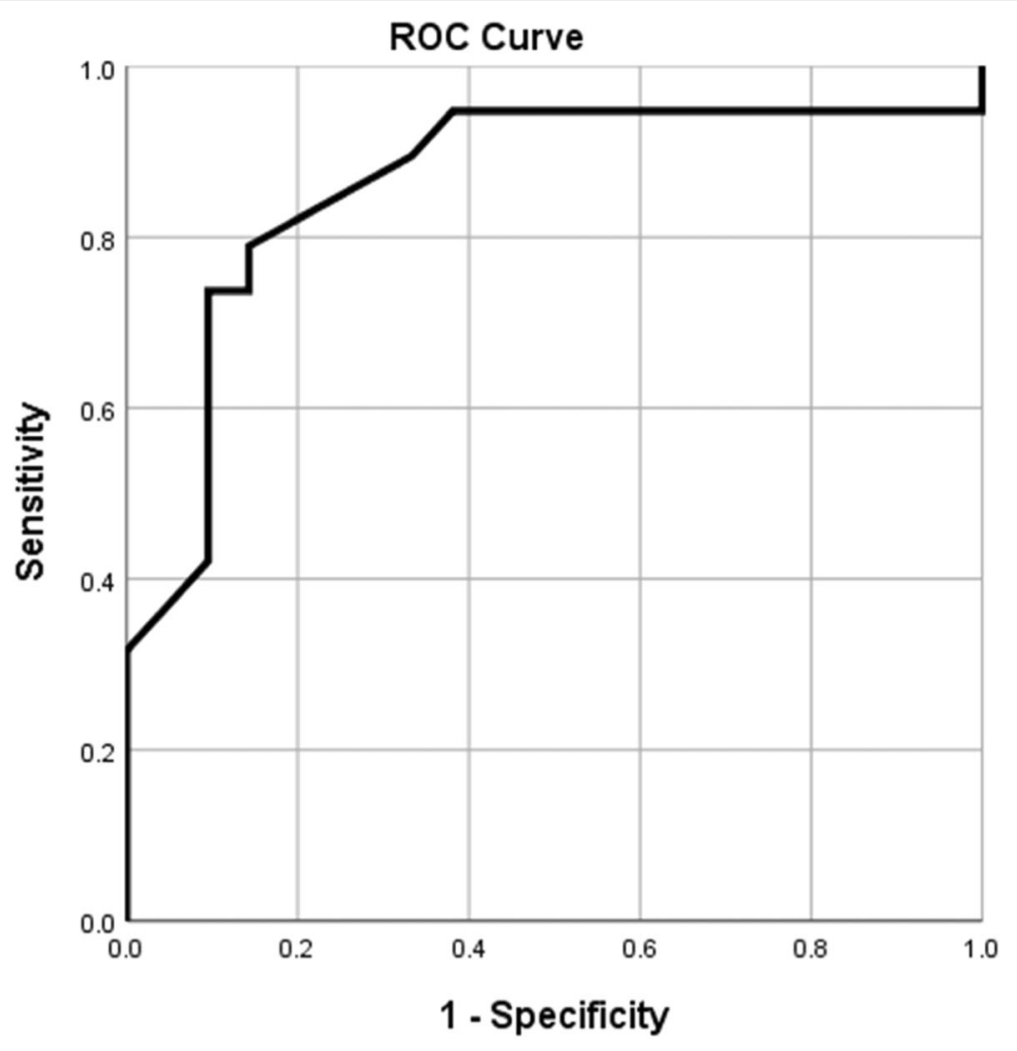

Diagonal segments are produced by ties.

Fig. 7 Results of receiver operating curves for ADC values in distinguishing non-viable and viable treat lesions groups

occur within tumors, where it permits easy evaluation of the whole tumor [18].

In our study, we concluded that diffusion-weighted imaging is of great value in case of contra-indication to contrast administration. However, it cannot completely substitute for DCE MRI study assessment, and interpretation should always be made in conjunction with conventional sequences and dynamic study to avoid errors.

We faced in our study few limitations. As for example, obtaining proven histopathology reports in patients who carried out TACE was difficult because our patients were not proper candidates for lesion resection or liver transplantation.

Another limitation in our study was the relatively small-sized sample diminishing the statistical analysis power. Small-sized sample could be attributed to the strict inclusion and exclusion criteria carried out for this research.

Also, we faced in DWI few technical difficulties in few treated lesions located along the upper portions of superior hepatic segments close to the diaphragmatic copula which are very sensitive to respiratory motion as well as pulsatile artifacts arising from the heart leading to relatively poor signal-to-noise ratio as well as degradation in spatial resolution. And during measuring ADC mapping values, we faced partial volume averaging effects and inevitable image noise leading to calculation errors. We tried to overcome these difficulties by placing the smallest possible ROI and having multiple measurements. So, we recommend more future studies with powerful magnetic gradient systems and new technical improvements to upgrade the sensitivity of DWI as well as image quality.

Table 5 Roc curve analysis revealed that ADC value was a significant discriminant factor in predicting non-viable from viable treat lesions groups ( $p$ value <0.001) with AUC 0.861 and 95\% confidence interval $(0.735-0.987$ )

\begin{tabular}{|c|c|c|c|c|c|c|c|c|c|c|}
\hline \multirow[t]{2}{*}{ Area } & \multirow[t]{2}{*}{ SE } & \multirow[t]{2}{*}{$P$ value } & \multicolumn{2}{|c|}{ 95\% confidence interval } & \multirow[t]{2}{*}{ Cutoff } & \multirow[t]{2}{*}{ Sensitivity } & \multirow[t]{2}{*}{ Specificity } & \multirow[t]{2}{*}{ PPV } & \multirow[t]{2}{*}{ NPV } & \multirow{2}{*}{$\begin{array}{l}\text { Overall } \\
\text { accuracy }\end{array}$} \\
\hline & & & Lower bound & Upper bound & & & & & & \\
\hline 0.861 & 0.064 & $<0.001$ & 0.735 & 0.987 & 1.35 & 78.9 & 85.7 & 83.33 & 81.82 & 82.50 \\
\hline
\end{tabular}


Table 6 Different indices of the dynamic MRI and the DWI

\begin{tabular}{lll}
\hline Statistics & Dynamic results (\%) & Diffusion results (uncertainty) as a positive interpretation (\%) \\
\hline Sensitivity & 100.00 & 52.63 \\
Specificity & 95.24 & 90.48 \\
Positive predictive value & 95.00 & 83.33 \\
Negative predictive value & 100.00 & 67.86 \\
Accuracy & 97.50 & 72.50 \\
\hline
\end{tabular}

As a result, dynamic MRI with subtracted images should be used together with DWI for optimum image interpretation in assessment of HCC viability post TACE.

Our study supports the follow-up post treatment algorithm suggested by LI-RADS v2018 to rule out falsenegative results caused by motion artifacts.

\section{Conclusions}

Our study showed that DCE MRI has 100\% level of sensitivity via application of LI-RADS v2018 diagnostic algorithmic approach while DWI alone has $52.63 \%$ level of sensitivity, yet it can enhance the diagnostic confidence of DCE MRI for post treatment response viability detection.

\section{Abbreviations}

ADC: Apparent diffusion coefficient; APHE: Arterial phase hyperenhancement; BCLC: Barcelona Clinic Liver Cancer; DCE: Dynamic contrast enhanced; DEBTACE: Drug-eluting bead transarterial chemoembolization; DWI: Diffusionweighted images; HCC: Hepatocellular carcinoma; IP: In-phase; LAVA: Liver acquisition with volume acceleration; LI-RADS: Liver Imaging Reporting and Data System; NPV: Negative predictive value; OP: Out-of-phase; PPV: Positive predictive value; ROC: Receiver operating characteristic; ROI: Region of interest; SPSS: Statistical Package for the Social Sciences; TACE: Transarterial chemoembolization; TAE: Transarterial embolization

\section{Acknowledgements}

Our sincere thanks to all our professors and colleagues in the Radiology Department, Faculty of Medicine, Ain shams University and Maadi Military Hospital for their support.

\section{Authors' contributions}

TY put the idea of the study, is the editor of the manuscript, was responsible for the image interpretation, and performed the statistical analysis. SB participated in the design of the study, image interpretation, and data collection. NS participated in the design of the study, image interpretation, and data collection. AYA participated in the design of the study, image interpretation, and data collection. All authors read and approved the final manuscript.

\section{Funding}

Not applicable (no funding received for this study).

\section{Availability of data and materials}

All the datasets used and analyzed in this study are available with the corresponding author on reasonable request.

\section{Ethics approval and consent to participate}

Written informed consent was signed by all patients before the examination. The study was approved by the Research Ethics Committee (REC) of the Faculty of Medicine, Ain Shams University, FWA 00017585 (approval number R1/2016), and experiments were conducted in accordance with the ethical guidelines.

\section{Consent for publication}

All patients included in this research were fully conscious and older than 16 years old and gave written informed consent to publish the data contained within this study.

\section{Competing interests}

The authors declare that they have no competing interests.

\section{Author details}

'Military Medical Academy, Heliopolis, Egypt. ²Faculty of Medicine, Ain Shams University, Cairo, Egypt.

Received: 12 August 2019 Accepted: 18 December 2019

Published online: 30 December 2019

\section{References}

1. Ren $A H$, Zhao PF, Yang DW et al (2019) Diagnostic performance of MR for hepatocellular carcinoma based on LI-RADS V2018, compared with v2017. J Magn Reson Imaging 50:746-755

2. Kulik LM, Chokechanachaisakul A (2015) Evaluation and Management of Hepatocellular Carcinoma. Clin Liver Dis 19:23-43

3. Van Beers, B.E., Daire, et al. 2015. Erratum to "New imaging techniques for liver diseases"[J Hepatol 2015; 62: 690-700]. J Hepatol, 63(3), p.774

4. Raoul JL, Forner A, Bolondi L et al (2019) Updated use of TACE for hepatocellular carcinoma treatment: how and when to use it based on clinical evidence. Cancer Treat Rev 72:28-36

5. Ebraheem Ebeed A, Abd El-hamied Romeih M, Mohamed Refat M et al (2017) Role of dynamic contrast-enhanced and diffusion weighted MRI in evaluation of hepatocellular carcinoma after chemoembolization. Egypt J Radiol Nucl Med 48:807-815

6. Kielar A, Fowler K, Lewis S et al (2018) Locoregional therapies for hepatocellular carcinoma and the new LI-RADS treatment response algorithm. Abdom Radiol 43:218-230

7. ElSaid NAE, Kaddah et al (2016) Subtraction MRI versus diffusion weighted imaging: which is more accurate in assessment of hepatocellular carcinoma after trans arterial chemoembolization (TACE)? Egyp J Radiol Nucl Med 47(4):1251-1264

8. Elsayes KM, Hooker JC, Agrons MM et al (2017) 2017 version of LI-RADS for CT and MR imaging: an update. RadioGraphics 37:1994-2017

9. Merkle EM, Zech et al (2016) Consensus report from the 7th international forum for liver magnetic resonance imaging. Eur Radiol 26(3):674-682

10. Galen RS (1980) Predictive value and efficiency of laboratory testing. Pediatr Clin N Am 27:861-869

11. Chan YH (2003) Biostatistics 103: qualitative data - tests of independence. Singap Med J 44:498-503

12. Chan YH (2003) Biostatistics 102: quantitative data--parametric \&amp; nonparametric tests. Singap Med J 44:391-396

13. Afifi AH, Naguib et al (2016) Diffusion weighted magnetic resonance imaging in assessment of hepatocellular carcinoma after chemoembolization. Egypt J Radiol Nucl Med 47(1):61-71 Elsevier B.V

14. Osama RM et al (2013) Role of dynamic contrast-enhanced and diffusion weighted MRI in evaluation of necrosis of hepatocellular carcinoma after chemoembolization. Egypt J Radiol Nucl Med 44(4):737-746 Elsevier B.V.

15. Yu JS et al (2009) Added value of diffusion-weighted imaging in the MRI assessment of perilesional tumor recurrence after chemoembolization of hepatocellular carcinomas. J Magn Reson Imaging 30(1):153-60

16. Goshima S et al (2008) Evaluating local hepatocellular carcinoma recurrence post-transcatheter arterial chemoembolization: is diffusion-weighted MRI reliable as an indicator? J Magn Reson Imaging 27(4):834-39 
17. Tantawy HI, Mohamed FF (2016) Diagnostic value of apparent diffusion coefficient (ADC) in evaluating hepatocellular carcinomas post transcatheter arterial chemoembolization and radiofrequency ablation. Egypt J Radiol Nucl Med 47(3):699-706

18. Yousef, M. I., Refaat, M. M. and Faheem, et al. (2017) 'Role of diffusionweighted magnetic resonance imaging in the evaluation of hepatocellular carcinoma response to transcatheter arterial chemoembolization using drug eluting beads; correlation with dynamic MRI', Egyptian Journal of Radiology and Nuclear Medicine. Egypt Soc Radiol Nucl Med, 48(4), pp. 817-824

\section{Publisher's Note}

Springer Nature remains neutral with regard to jurisdictional claims in published maps and institutional affiliations.

Submit your manuscript to a SpringerOpen ${ }^{\circ}$ journal and benefit from:

- Convenient online submission

- Rigorous peer review

- Open access: articles freely available online

High visibility within the field

- Retaining the copyright to your article

Submit your next manuscript at $\boldsymbol{\nabla}$ springeropen.com 